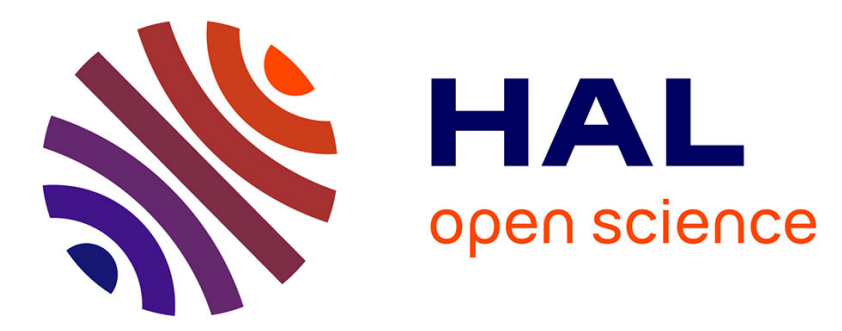

\title{
Source separation on hyperspectral cube applied to dermatology
}

\author{
Jhimli Mitra, Romuald Jolivot, Franck S. Marzani, Pierre Vabres
}

\section{To cite this version:}

Jhimli Mitra, Romuald Jolivot, Franck S. Marzani, Pierre Vabres. Source separation on hyperspectral cube applied to dermatology. SPIE Medical Imaging meeting, Feb 2010, San Diego, United States. pp.762431-1, 762431-11. hal-00638586

\section{HAL Id: hal-00638586 https://hal.science/hal-00638586}

Submitted on 7 Nov 2011

HAL is a multi-disciplinary open access archive for the deposit and dissemination of scientific research documents, whether they are published or not. The documents may come from teaching and research institutions in France or abroad, or from public or private research centers.
L'archive ouverte pluridisciplinaire HAL, est destinée au dépôt et à la diffusion de documents scientifiques de niveau recherche, publiés ou non, émanant des établissements d'enseignement et de recherche français ou étrangers, des laboratoires publics ou privés. 


\title{
Source Separation on HyperSpectral Cube Applied to Dermatology
}

\author{
Mitra J., Jolivot R., Marzani F.S., Vabres P. \\ Le2i, UMR CNRS 5158, UFR Sc. \& Tech., University of Burgundy BP 47870, 21078 Dijon \\ Cedex, France
}

\begin{abstract}
This paper proposes a method of quantification of the components underlying the human skin that are supposed to be responsible for the effective reflectance spectrum of the skin over the visible wavelength. The method is based on independent component analysis assuming that the epidermal melanin and the dermal haemoglobin absorbance spectra are independent of each other. The method extracts the source spectra that correspond to the ideal absorbance spectra of melanin and haemoglobin. The noisy melanin spectrum is fixed using a polynomial fit and the quantifications associated with it are reestimated. The results produce feasible quantifications of each source component in the examined skin patch.
\end{abstract}

Keywords: Source separation, Spectral reflectance, Non-Gaussianity, Kurtosis, Independent Component Analysis.

\section{INTRODUCTION}

Human skin has a complex structure with variable geometry. In most cases skin diseases are diagnosed by dermatologists with naked eye. However, in certain cases, for example differentiating melanoma (skin cancer) with nevus (benign dark patch) becomes difficult under normal lighting conditions. In the field of medicine, there have been significant researches using invasive and non-invasive techniques to diagnose skin diseases. Optical reflectance properties of skin play important roles in diagnosis of skin diseases using non-invasive techniques.

Optical reflectance of skin is primarily contributed by the upper (epidermis) and the lower (dermis) layers. The epidermis contains melanin as its primary component, while the dermis contains the blood vessels carrying the oxygenated blood (oxy-haemoglobin, $\mathrm{HbO}_{2}$ ) and the deoxygenated blood (deoxy-haemoglobin, $\mathrm{Hb}$ ) respectively. In normal lighting conditions it is not possible to characterise the components such as melanin, $\mathrm{Hb}_{\mathrm{b}} \mathrm{an} \mathrm{HbO}_{2}$. However, it might be possible to know them by acquiring images at different wavelengths and generating an overall reflectance spectrum of the skin.

The ASCLEPIOS system developed by the M2D+ team of Le2i, exploits the spatial resolution of the skin image along with the reflectance spectrum over different wavelengths. The device captures seven gray-level images of the skin patch with seven different interference filters at different wavelengths. These images constitute a multispectral image. ${ }^{1}$ Thus the vector at each pixel comprises of seven values. The multispectral image is then reconstructed to produce reflectance values for each pixel over thirty-six wavelengths. These reflectance values constitute the hyperspectral cube. ${ }^{2}$

The focus of this work has been to estimate the characteristic reflectance spectrum of the skin components and also to quantify the amount of a component spectrum in the total reflectance. Our algorithm accepts reconstructed reflectance images to estimate the reflectance spectrum of the source components and generates images with effective quantification of the source components.

The rest of the paper has been organised in the following manner. Section 2 discusses the common methods to decompose mixed multivariate data into its source components and the method applied in our work. Section 3 discusses the results with real skin images followed by conclusion and bibliography in the subsequent sections.

Further author information: (Send correspondence to J. Mitra or Dr. F.S. Marzani.)

J. Mitra: E-mail: jhimlimitra@yahoo.com,

R. Jolivot: E-mail: romuald.jolivot@u-bourgogne.fr,

F.S. Marzani: E-mail: franck.marzani@u-bourgogne.fr, Telephone: +33(0)380396333

P. Vabres: E-mail: pierre.vabres@u-bourgogne.fr 


\section{METHODS OF SOURCE SEPARATION}

Source separation of mixed signals has been a challenging problem since eighties. The classical problem in this domain is the cocktail-party problem where a number of people are talking simultaneously. It is possible for a human being to follow one particular discussion, because a human brain can perform real-time processing of the mixed signal to separate into its respective components. A lot of work has been done in this direction based on Principal Component Analysis (PCA) and Independent Component Analysis (ICA).

PCA is a classical statistical technique that aims to reduce redundancy from a multivariate measurement by reducing the number of variables while maximally representing the original signal. PCA takes into account the correlation of the data elements and performs a linear projection of the data from the higher dimension to a lower dimension by considering the eigen vectors and the eigen values obtained from the single-valued-decomposition of the covariance matrix of the data elements. However, this approach was extended to ICA to formulate a statistically stable model that provides sources that are more relevant along with a quantification of the sources in the mixed signal.

ICA assumes a model where the mixed signal is considered to be a linear mixture of sources with some noise that have been caused by the sensors used for the measurement. The method basically tries to find components that are statistically independent and non-Gaussian except if only one component is Gaussian.

ICA can be performed by maximising non-Gaussianity. The classical method in maximisation of such measure is the maximization of the kurtosis of the source components. The fourth-order cumulant or the kurtosis of a Gaussian component is zero. However, if the distribution of the source components is super-Gaussian or subGaussian, kurtosis is either positive or negative respectively. ${ }^{3}$

Another measure of non-Gaussianity is the negentropy. Entropy is an important concept in information theory. A Gaussian distribution has the maximum entropy among any random distribution. Whereas, a distribution that is spiky has the smaller entropy. Differential entropy or negentropy can be defined as the difference of a random distribution from Gaussian distribution entropy. Therefore, maximizing negentropy would yield source components that are non-Gaussian. However, this method is computationally complicated due to the choice of the non-linear function for approximation of negentropy. ${ }^{4}$

If the source components are considered to be independent as is normally the case with ICA, then minimizing the mutual information of the components would be a criterion for estimating the same. Mutual information is the dependence of the components on one another and can be represented as the difference of negentropy of one component from the sum of the negentropies of other source components.

Since the source components are independent, estimating maximum likelihood can be another approach to estimate the components. ${ }^{5}$ If the density distribution of the components is simple with very few parameters to estimate, then the maximum likelihood approach is a good choice. However, it is often difficult to make an assumption about the components' density distribution. Therefore, a rough estimation about the form of the distribution does not always yield good solution. Also the complexity increases in parameter estimation if the density is not a simple one.

An algebraic method for source separation is non-negative matrix factorization proposed by Lee and Seung. ${ }^{6}$ In this approach, the coefficients of the source component spectrum as well as the quantifications or mixing values of the sources in the mixed signal are constrained to be strictly positive. The method was applied by Sajda et al. ${ }^{7}$ for recovering constituent spectra from data of 3D chemical shift imaging of brain. This method however, does not assume that the source signals are independent which can be a fair consideration in certain cases.

For estimation of skin components, Tsumura et al. ${ }^{8,9}$ adopted an approach based on PCA and ICA. The authors used a single colour image or the reflectance spectra of skin over three wavelengths (red, green and blue). They estimated only two components as melanin and haemoglobin from the skin reflectance by reducing the dimensionality of the data.

In our work, we chose a method where, we assume that few source components like melanin and haemoglobin are independent and employ the kurtosis maximization of the source components as a measure of non-Gaussianity and a fixed-point algorithm rather than the gradient algorithm since the convergence of the former is cubic and 
faster. The melanin spectrum obtained using ICA method is normally noisy and there have been researches trying to validate the obtained melanin spectrum. ${ }^{10}$ However, we adopt a new method to fix the melanin spectrum from the noisy spectrum. In the following sub-section the method employed for source separation related to our problem has been discussed in detail.

\section{Independent Component Analysis with Principal Component Analysis}

Hyvärinen et al. ${ }^{3}$ proposed several techniques for Independent Component Analysis (ICA) for decomposing multivariate data into its independent source components. A very important contribution of their works on ICA is the Fast ICA algorithm. We have applied the Fast ICA for our problem of skin component separation to estimate the independent components using a measure of non-Gaussianity. In our work melanin and haemoglobin might be assumed as independent source components.

In ICA, an important criterion is, if there are $m$ observed random variables, $\left[v_{1}, v_{2}, \ldots, v_{m}\right]^{T}$ forming $\mathbf{V}$, then, there are $l$ source components $\left[s_{1}, s_{2}, \ldots, s_{l}\right]^{T}$ forming $\mathbf{S}$ where, $l \leq m .{ }^{3,11}$ The linear relationship between the mixture and the independent sources can be given by

$$
\mathbf{V}=\mathbf{A S}
$$

Here, $\mathbf{A}$ is the $m \times l$ mixing matrix of full-rank.

The choice of ICA for this problem is due to its ability to find non-Gaussian components. The problem of finding the mixing matrix $\mathbf{A}$ and the non-Gaussian components $s_{i}, i=1,2, \ldots, l$, is solved by defining that $s_{i}$ has unit variance and somehow reducing the task of estimating the full-rank matrix $\mathbf{A}$ in to a square matrix.

In our experiment, it is required to estimate two independent components out of $m$ mixtures (reflectance data of pixels). Therefore, it is required to find out the two best representations out of $m$ representations. This is achieved by Principal Component Analysis (PCA). PCA not only reduces the dimensionality but also does a sphering or prewhitening of the data so that the task of finding the mixing matrix reduces to the task of estimating a square orthogonal matrix.

The observed data $\mathbf{V}$ is linearly transformed using PCA such that

$$
\mathbf{X}=\mathbf{M V}=\mathbf{M A S}=\mathbf{B S}
$$

where, $\mathbf{B}=\mathbf{M A}$ is the orthogonal mixing matrix with the assumptions that the components have unit variance.

Therefore,

$$
E\left\{\mathbf{X X}^{T}\right\}=\mathbf{B} E\left\{\mathbf{S S}^{T}\right\} \mathbf{B}^{T}=\mathbf{B} \mathbf{B}^{T}=\mathbf{I}
$$

The problem is now reduced to the estimation of the orthogonal mixing matrix $\mathbf{B}$. The columns of the matrix $\mathbf{B}$ are denoted by $b_{i}$ and the $i^{t h}$ source component can be computed using $\mathbf{X}$ by equation 4 .

$$
s_{i}=\left(b_{i}\right)^{T} \mathbf{X}=\mathbf{w}^{T} \mathbf{X} .
$$

Whitening of the observed data $\mathbf{V}$ into $\mathbf{X}$ by PCA reduces the dimensionality of $\mathbf{V}$ from $m$ to $l$ and also ensures that the transformed data $\mathbf{X}$ lie within a unit sphere, i.e. the covariance of $\mathbf{X}$ is equal to identity. The mean of the observed data is removed to center the data about zero and then the covariance of the transpose of the observed data is computed. The significance of the transpose is that the required covariance matrix $\mathbf{C}$ is of dimension $m \times m$ that results in the sample size of the obtained source components' spectra to be the same as the sample size of the observed reflectance data of each mixed pixel. Eigen decomposition of the covariance matrix yields a diagonal matrix $\mathbf{D}$ of eigen-values in ascending order and a matrix $\mathbf{E}$ whose columns form the corresponding eigen-vectors associated with the eigen-values such that the relation in Eq. (5) holds. 


$$
\mathbf{C}=\mathbf{E D E}^{T} .
$$

The matrices $\mathbf{E}$ and $\mathbf{D}$ both are $m \times m$. To reduce the dimensions of $\mathbf{E}$ to $m \times l$ and $\mathbf{D}$ to $l \times l$ respectively, where, $l$ is the number of source components to be extracted, the highest $l$ eigen-values and their corresponding columns of $\mathbf{E}$ are retained. The new whitened and reduced dimensioned data is represented in the reduced subspace of $\tilde{\mathbf{E}}$ and $\tilde{\mathbf{D}}$.

In our experiment, $l=2$ as there are two source components to be extracted. The whitening matrix $\mathbf{M}$ can be represented as in Eq. (6).

$$
\mathbf{M}=\tilde{\mathbf{D}}^{-1 / 2} \tilde{\mathbf{E}}^{T} .
$$

Therefore, the whitened data in the reduced subspace is

$$
\mathbf{X}=\mathbf{M V}=\tilde{\mathbf{D}}^{-1 / 2} \tilde{\mathbf{E}}^{T} \mathbf{V}
$$

From Eq. (2) we have the following transformation.

$$
\mathbf{A}=\mathbf{M}^{-1} \mathbf{B} .
$$

Where, $\mathbf{M}^{-1}$ is the dewhitening matrix and is represented as

$$
\mathbf{M}^{-1}=\left(\tilde{\mathbf{D}}^{-1 / 2} \tilde{\mathbf{E}}^{T}\right)^{-1}=\tilde{\mathbf{E}} \tilde{\mathbf{D}}^{1 / 2} .
$$

Kurtosis maximization as a measure of non-Gaussianity has been a classical technique to estimate the independent components. Due to the non-Gaussianity of the source components, kurtosis maximisation of the source components has been employed in this work. However, it is to be noted at this point that maximisation of kurtosis is basically maximising the absolute value of the kurtosis. This enables to extract both sub-Gaussian and super-Gaussian components with negative and positive kurtosis respectively. Kurtosis of a random variable $y$ with zero mean can be defined as

$$
\operatorname{kurt}(y)=E\left\{y^{4}\right\}-3\left(E\left\{y^{2}\right\}\right)^{2} .
$$

Aiming to maximise the non-Gaussianity i.e. the kurtosis of the independent components another constraint holds i.e. $E\left\{y^{2}\right\}=1$. Which implies that, since the components are independent, they have unit variance. Therefore, using Eq. (4) the independent components can be estimated by maximisation of the kurtosis $k u r t\left(\mathbf{w}^{T} \mathbf{X}\right)$ under the constraint $E\left\{\left(\mathbf{w}^{T} \mathbf{X}\right)^{2}\right\}=\|\mathbf{w}\|^{2}=1$, that follows from Eq. (11) as $\mathbf{X}$ is already whitened.

$$
E\left\{y^{2}\right\}=E\left\{\mathbf{w}^{2}\right\} E\left\{\mathbf{X}^{2}\right\}=E\left\{\mathbf{w}^{2}\right\}=1 .
$$

Therefore, in the optimization problem we seek for a vector $\mathbf{w}$ such that the linear combination $\mathbf{w}^{T} \mathbf{X}$ has the maximum non-Gaussianity.

The cost function for the optimization is defined as

$$
J(\mathbf{w})=E\left\{\left(\mathbf{w}^{T} \mathbf{X}\right)^{4}\right\}-3\left(E\left\{\left(\mathbf{w}^{T} \mathbf{X}\right)^{2}\right\}\right)^{2} .
$$

The gradient of the cost function with respect to $\mathbf{w}$ is shown in Eq. (13).

$$
\frac{\delta J(\mathbf{w})}{\delta \mathbf{w}}=E\left\{\mathbf{X}\left(\mathbf{w}^{T} \mathbf{X}\right)^{3}\right\}-3\|\mathbf{w}\|^{2} \mathbf{w} .
$$


At stable point, this gradient becomes equal to zero and no change occurs with $\mathbf{w}$ if the gradient is added to it. Therefore, using fixed-point iteration scheme, the update for the new value of $\mathbf{w}(t)$ can be made using the function of $\mathbf{w}(t-1)$. Here, $t$ is the iteration number.

$$
\mathbf{w}(t)=E\left\{\mathbf{X}\left(\mathbf{w}(t-1)^{T} \mathbf{X}\right)^{3}\right\}-3 \mathbf{w}(t-1)
$$

The iteration continues until the direction of the old and new values of $\mathbf{w}$ are the same; i.e. the absolute value of the dot product of the two vectors is almost equal to one. After each update, w is normalised to keep the vector within a unit sphere as in Eq. (15).

$$
\mathbf{w}=\mathbf{w} /\|\mathbf{w}\|
$$

$\mathbf{w}$, is a vector representing a column of the mixing matrix $\mathbf{B}$, therefore, only one source component is obtained using this method. The other components are obtained using the Gram Schmidt deflation approach ${ }^{12}$ where a matrix $\overline{\mathbf{B}}$ is formed with the already found $\mathbf{w}$ vectors and the current estimate of $\mathbf{w}(t)$ is projected back into the orthogonal space of $\overline{\mathbf{B}}$ before the normalisation step as in Eq. (16).

$$
\mathbf{w}(t)=\mathbf{w}(t)-\overline{\mathbf{B}} \overline{\mathbf{B}}^{T} \mathbf{w}(t) .
$$

This step of decorrelation ensures that we do not obtain the same independent component. The dewhitened matrix $\mathbf{A}$ is obtained using Eq. (8).

The noisy melanin spectrum is fixed using a straight line fit that closely corresponds to the ideal spectrum and the associated mixing vector of melanin is reestimated. The obtained source spectra and their mixing quantities ideally form a linear model to produce the observed mixed signals. Therefore, the proportion of haemoglobin formed by the haemoglobin spectrum and its associated quantifications is subtracted from the mixture to leave the melanin part as residue. The fixed melanin spectrum is used to reestimate the quantifications of melanin from the residual mixture. The quantifications of melanin is estimated using least squares optimization. Fig. 1 shows the obtained melanin and haemoglobin spectra before and after polynomial fit of melanin.

\section{RESULTS AND DISCUSSIONS}

The skin reflectance values obtained from the reconstruction process for each pixel is in terms of percentage values over 36 wavelengths sampled equally over $430 \mathrm{~nm}$ to $780 \mathrm{~nm}$. Therefore, we have 36 sets of reflectance planes reconstructed from a multispectral image with seven filters acquired by the device ASCLEPIOS within a visible range. Each pixel is an observation in the form of a vector of 36 dimensions. We have the prior knowledge about the absorbance spectrum of the underlying skin components from the data by Jacques and Prahl. ${ }^{13,14}$

The absorbance spectrum of oxy-haemoglobin has a characteristic 'W' shape between $430-560 \mathrm{~nm}$. The deoxy-haemoglobin absorbance spectrum almost has the same shape as the former, except for a less prominent 'W'. The melanin spectrum is a flat decaying curve. The reflectance spectra are assumed to be inverse logarithm of absorbance spectra of the components. Therefore, the characteristic reflectance spectra of human skin components are shown in fig. 2 .

The method of ICA was applied on the skin reflectance data of a specific pathology known as 'café-au-lait' meaning "coffee with milk". These are hyperpigmented lesions found in skin or iris due to increased content of melanin. This method considers the two independent component spectra as haemoglobin and melanin and extracts the same along with the mixing matrix whose columns are normalised and represent quantifications of each independent component.

The quantifications are converted into gray scale images by normalisation of the obtained values within a range of $[0-255]$. The order of extraction of the source components as haemoglobin or melanin determines whether the first column of the mixing matrix represents the respective quantification of haemoglobin and the second column for melanin or vice versa. The advantage of this ICA method is that the convergence is often 


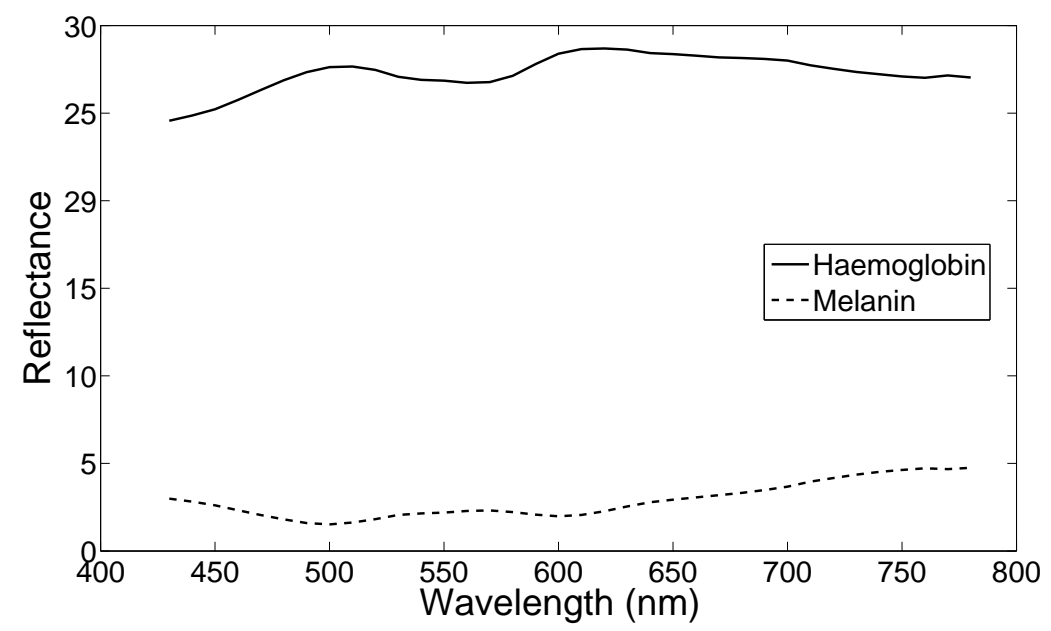

(a)

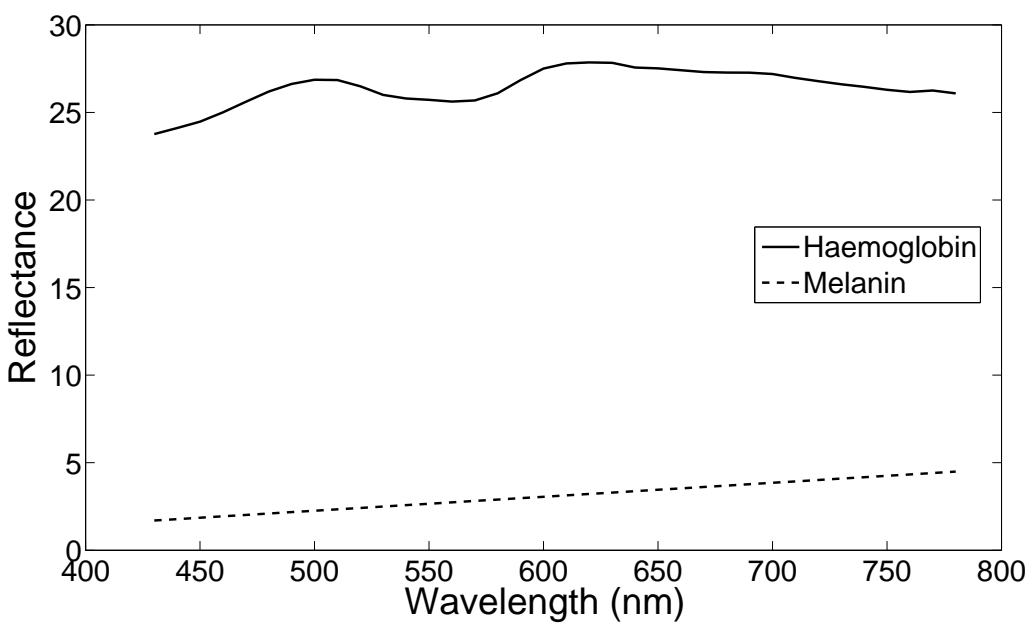

(b)

Figure 1. (a) Melanin and haemoglobin spectra obtained before fitting, (b) Haemoglobin and melanin spectra after polynomial fit.

in 5-6 steps; therefore, it is computationally less expensive than the gradient-descent algorithms. Also, it was shown by Hyvärinen ${ }^{4}$ that the algorithm always converges and the convergence is cubic. However, a significant amount of computation might be needed to compute the covariance matrix if the size of skin patch is too large.

The sample patch that was processed comprised of portions of lightly pigmented 'café au lait' spot and normal skin on the left and right parts of the original image respectively fig. 3(a). Histogram analysis was done with the obtained quantification images. Therefore, from fig. 3(c) and fig. 3(d) it is evident that the melanin is more quantified on the left part of the image with black spots embedded that characterise the milky spots in the region having less melanin. Fig. 3(e) shows an almost uniform quantification of haemoglobin over the same patch and therefore fig. 3(f) has an almost centered peak.

Additionally, almost homogeneous patches from only normal part and only 'café au lait' spot were processed separately. The spectra obtained for haemoglobin and corrected melanin for all the patches closely resemble the ideal spectra. The patch in fig. 4(a) was taken from the normal part of the skin. Fig. 4(f) shows a little right skewed peak than fig. 4(d) which suggests that the haemoglobin quantification in normal parts of skin is more than the melanin quantification for Caucasian skins. 


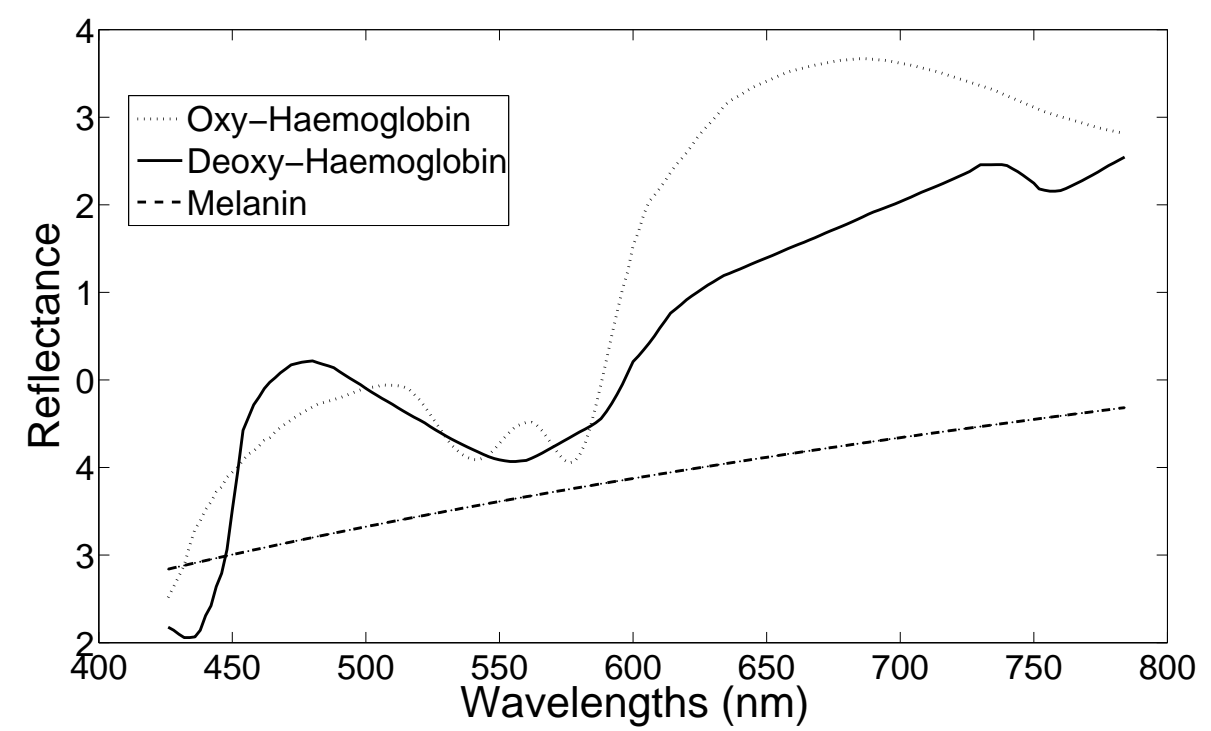

Figure 2. Ideal Reflectance Spectra for Oxy-haemoglobin, Deoxy-haemoglobin and Melanin

The histograms in fig. 5(f) and fig. 5(d) show single mode peaks that suggest that both have almost uniform quantifications. The peak in fig. 5(f) is almost center skewed, while that in fig. 5(d) is right skewed. This suggests that the patch has more quantification of melanin and less of haemoglobin as the patch is taken only from the café-au-lait part of the skin.

\section{CONCLUSIONS AND FUTURE WORKS}

A technique to separate various components underlying the skin and that are responsible for the effective skin reflectance spectra have been proposed and implemented. An effective technique that is based on ICA and PCA have been implemented to separate the possible independent components like melanin and haemoglobin and estimate the quantifications associated with each of these components. The results obtained using this technique show feasible quantifications of melanin and haemoglobin when tested on a 'café-au-lait' patch that causes pigmentation only due to the excess of melanin. The test has been performed using the 'café-au-lait' spot of only one patient where the result is consistent on every execution. However, it is assumed that the algorithm will provide consistent and feasible results for all such melanin pigmentations. This method can also be used for quantification of haemoglobin that is very important in diagnosing erythema that is a 'reddish' inflammation of skin due to the dilation of blood vessels in the skin dermis.

The ICA estimation method can be extended in future for pixel-wise extraction of melanin and haemoglobin based on the reduction of reflectance spectrum of each pixel which might provide better pixel-wise quantifications. Non-linearities other than the fourth order cumulant can be used for better estimation of the components. The components oxy- and deoxy-haemoglobin are assumed to be dependent components; however, this assumption might not be correct as not much research have been done in this direction. Therefore, ICA method applied on the residual mixture after subtraction of the extracted melanin portion could yield components that could be assumed to be oxy- and deoxy-haemoglobin respectively. For further validation this method could be tested on a skin pathology called 'vitiligo' that is whitening of the skin due to absence of melanin.

Therefore, we may conclude that the system ASCLEPIOS along with this technique of source separation can make significant contribution to the research of non-invasive techniques for the diagnosis of skin diseases.

\section{ACKNOWLEDGMENTS}

I would like to thank my supervisors Dr. F. Marzani and Dr. P. Vabres for their guidance in understanding the subject. I would also like to extend my thanks to Mr. R. Jolivot and Mr. T. Decourselle of M2D+ team, Le2i 
for their constant support in achieving the results.

\section{REFERENCES}

[1] Mansouri, A., Marzani, F., Hardeberg, J., and Gouton, P., "Optical calibration of a multispectral imaging system based on interference filters," Journal of Optical Engineering 44, 027004.1-0270041.2 (February 2005).

[2] Marzani, F., Mansouri, A., Vabres, P., and Gouton, P., "Méthode pour l'analyse d'une zone de la peau," Tech. Rep. dépôt d'une demande de brevet FR 0951233, copropriétaires Université de Bourgogne, CNRS et CHU Dijon (February 2009).

[3] Hyvärinen, A., Karhunen, J., and Oja, E., [Independent Component Analysis], John Wiley, New York (2001).

[4] Hyvärinen, A., "Fast and robust fixed-point algorithms for independent component analysis," IEEE Trans. on Neural Networks 10(3) (1999).

[5] Cardoso, J.-F., "Infomax and maximum likelihood for blind source separation," IEEE Signal Proc. Letters 4(4) (1997).

[6] Lee, D. and Seung, H., "Learning the parts of objects by non-negative matrix factorization," Nature 401, 788-791 (1999).

[7] Sajda, P., Du, S., Brown, T., Parra, L., and Stoyanova, R., "Recovery of constituent spectra in 3d chemical shift imaging using non-negative matrix factorization," Proc. International Conference on Independent Component Analysis and Blind Signal Separation (ICA'2003), 71-76 (2003).

[8] Tsumura, N., Haneishi, H., and Miyake, Y., "Independent component analysis of skin color image," J. Opt. Soc. Am. A 16(9), 2167-2176 (1999).

[9] Tsumura, N., Haneishi, H., and Miyake, Y., "Independent component analysis of spectral absorbance image in human skin," Optical Review 7(6), 479-482 (2000).

[10] Xu, S., Ye, X., Wu, Y., Giron, F., Leveque, J. L., and Querleux, B., "Automatic skin decomposition based on single image," Computer Vision and Image Understanding 110, 1-6 (2008).

[11] Comon, P., "Independent component analysis-a new concept?," Signal Processing 36, 287-314 (1994).

[12] Hyvärinen, A. and Oja, E., "A fast fixed-point algorithm for independent component analysis," Neural Computation 9, 1483-1492 (1997).

[13] Jacques, S. L., "Optical absorption of melanin," Oregon Medical Laser Center Monthly news and articles on Biomedical Optics and Medical Lasers http://omlc.ogi.edu/spectra/melanin/index.html (1998).

[14] Prahl, S., "Optical absorption of hemoglobin," Oregon Medical Laser Center Monthly news and articles on Biomedical Optics and Medical Lasers http://omlc.ogi.edu/spectra/hemoglobin/index.html (1999). 


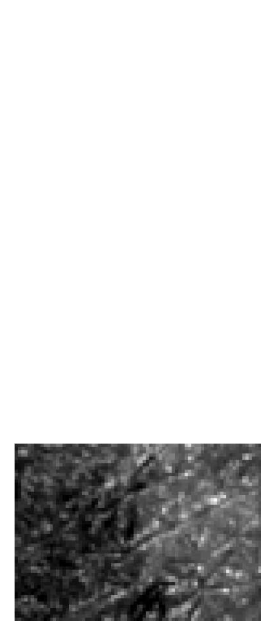

(a)

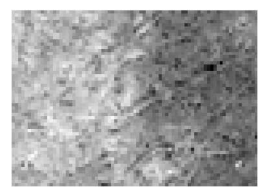

(c)

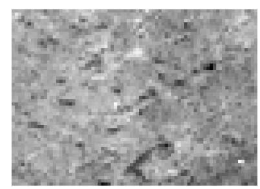

(e)

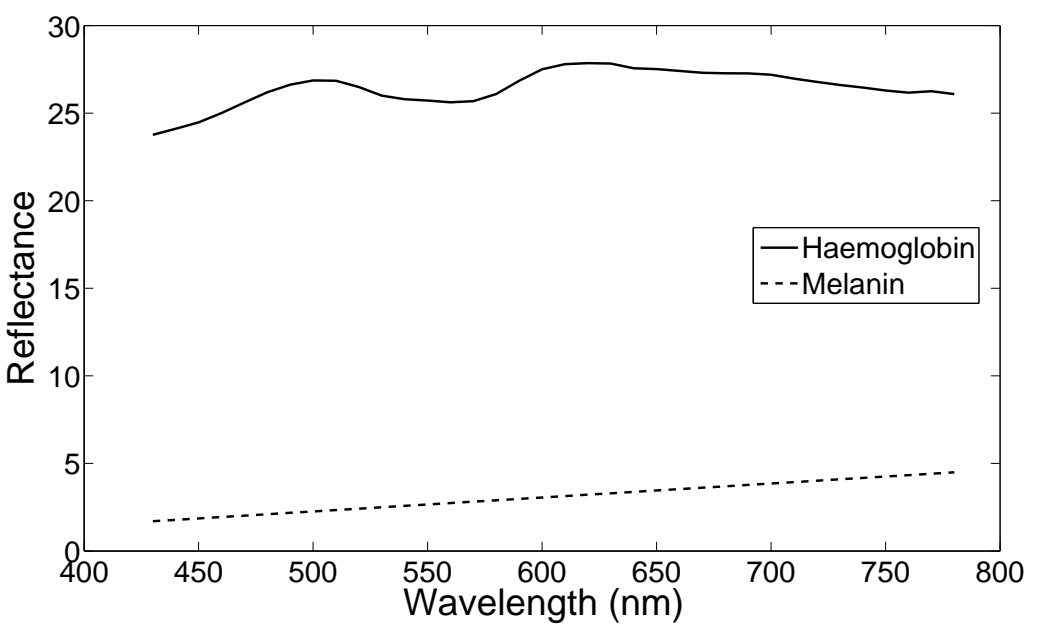

(b)

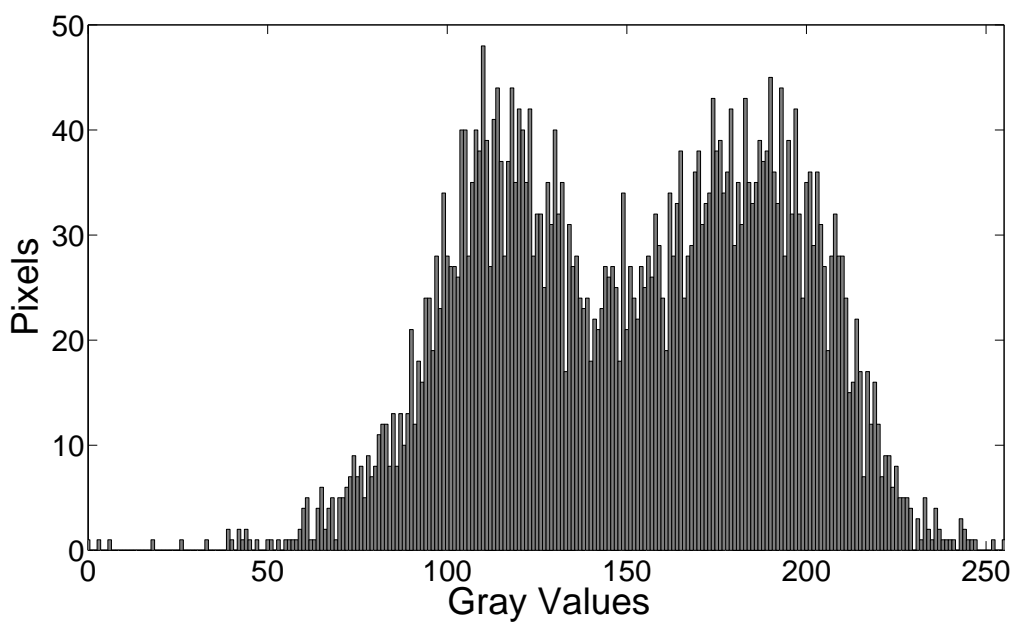

(d)

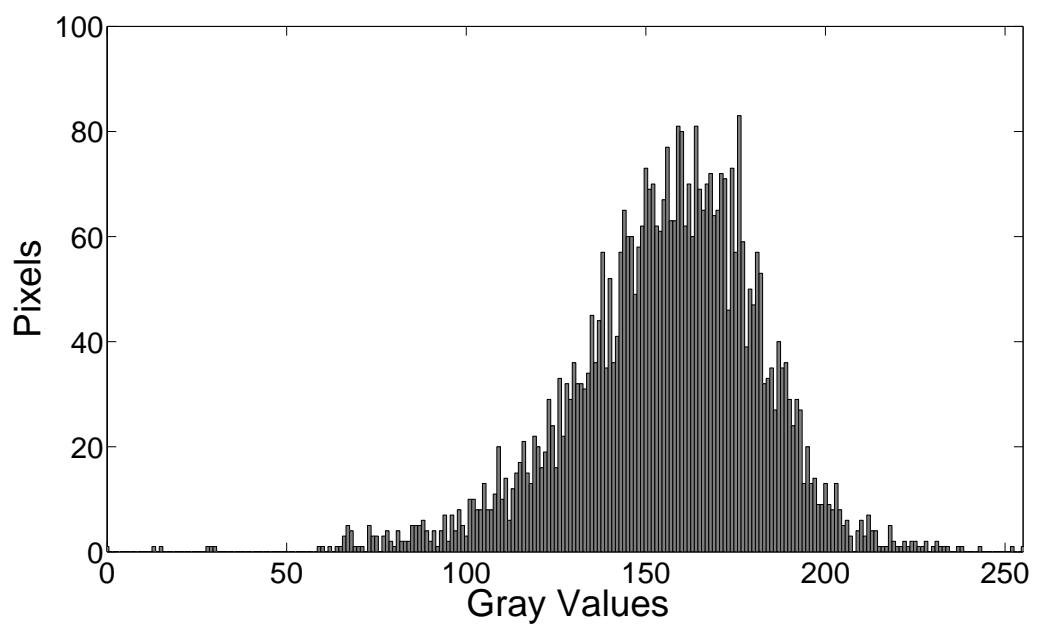

(f)

Figure 3. (a)Skin patch comprising both café-au-lait (left) and normal part (right), (b)Obtained source spectra, (c) Melanin quantification, (d) Histogram of melanin quantification, (e) Haemoglobin quantification, (f) Histogram of haemoglobin quantification 


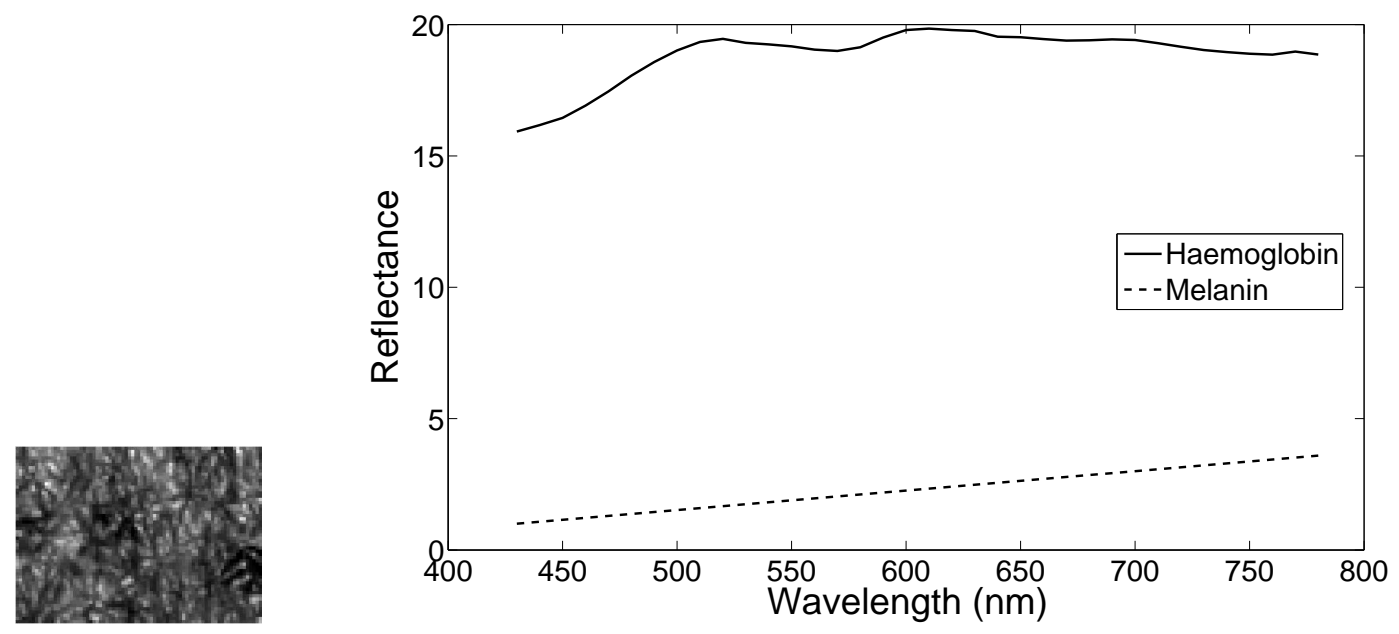

(a)

(b)

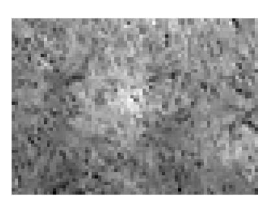

(c)

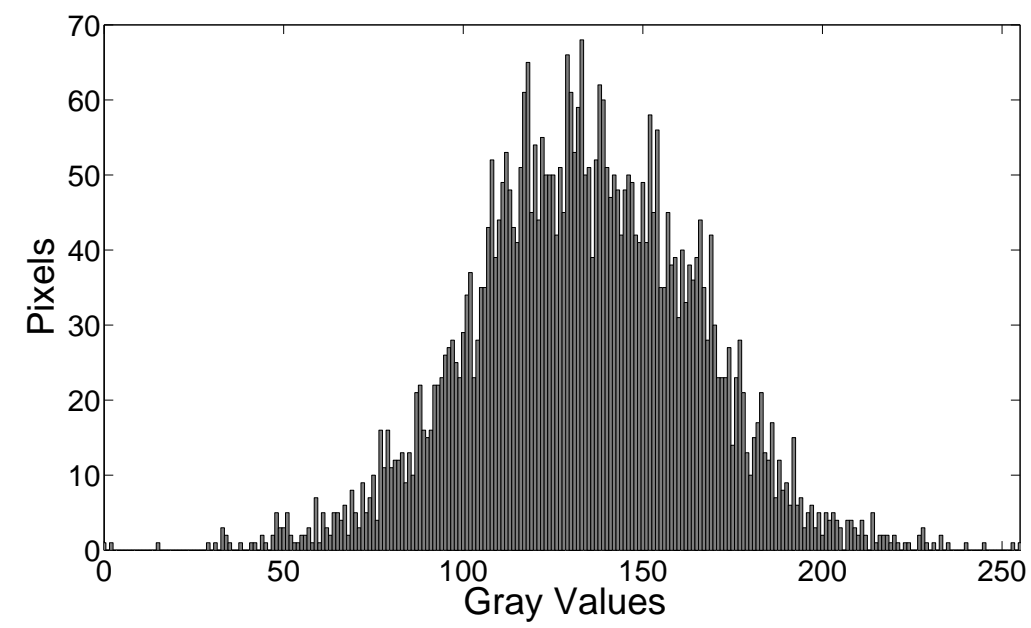

(d)

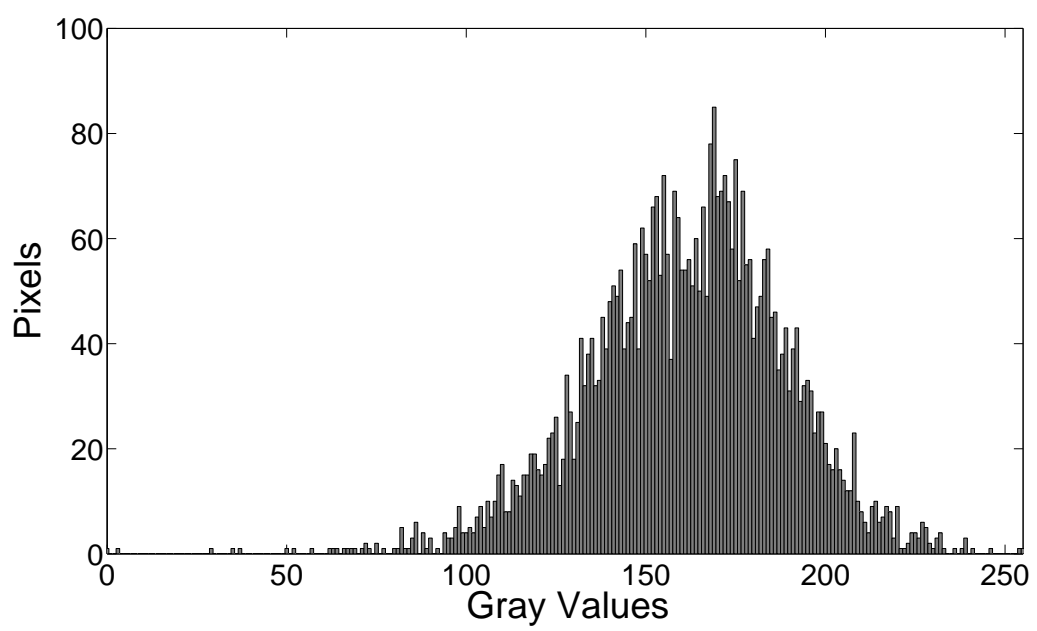

(f)

Figure 4. (a) Skin patch from normal part, (b) Obtained source spectra, (c) Melanin quantification, (d) Histogram of melanin quantification, (e) Haemoglobin quantification, (f) Histogram of haemoglobin quantification 


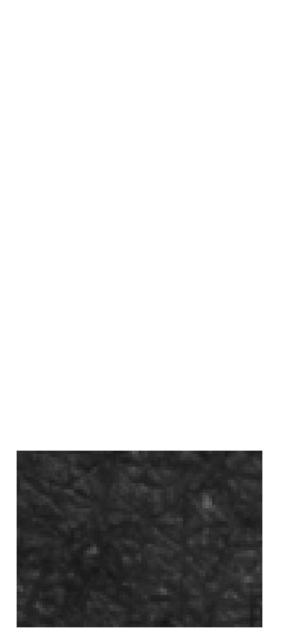

(a)

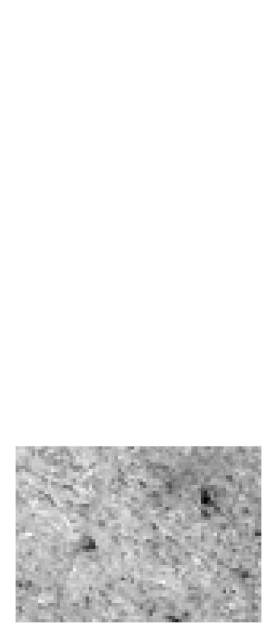

(c)

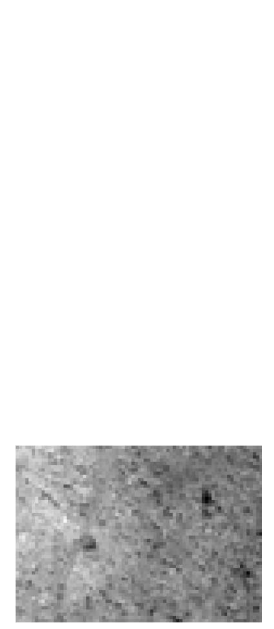

(e)

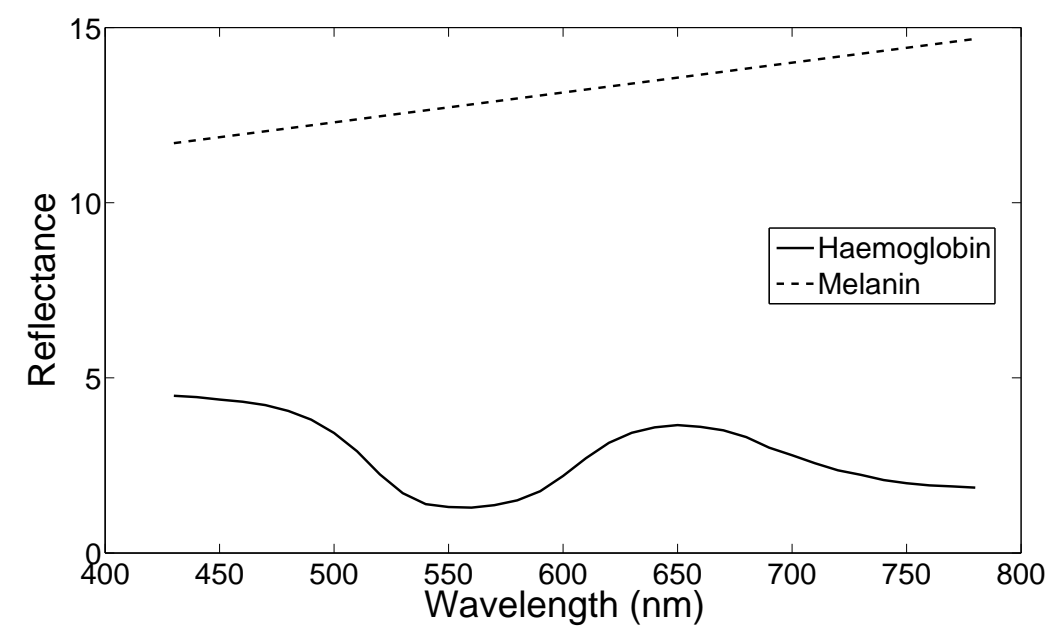

(b)

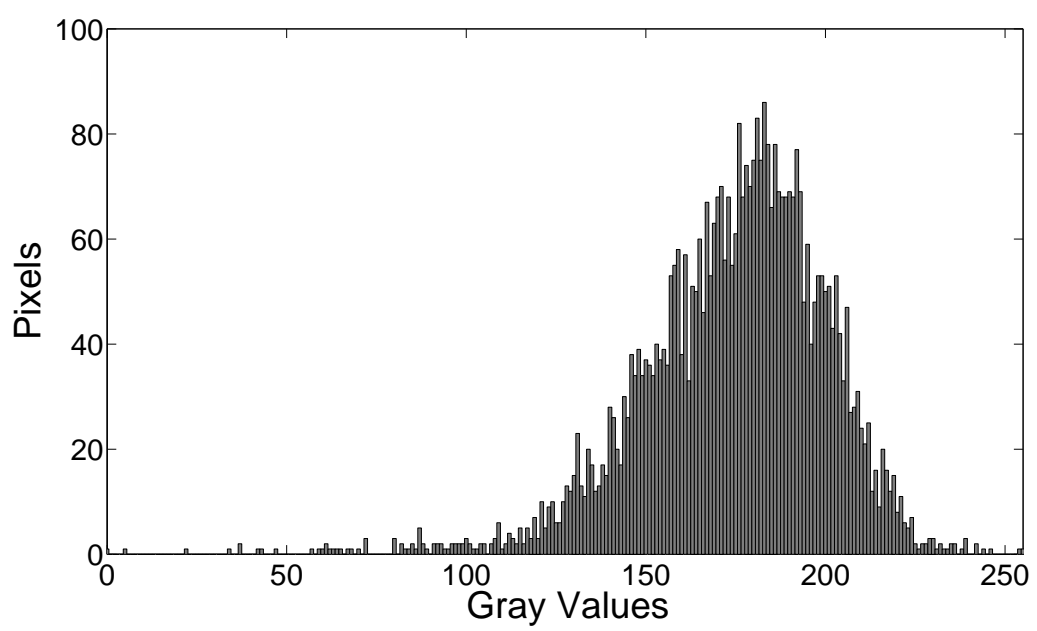

(d)

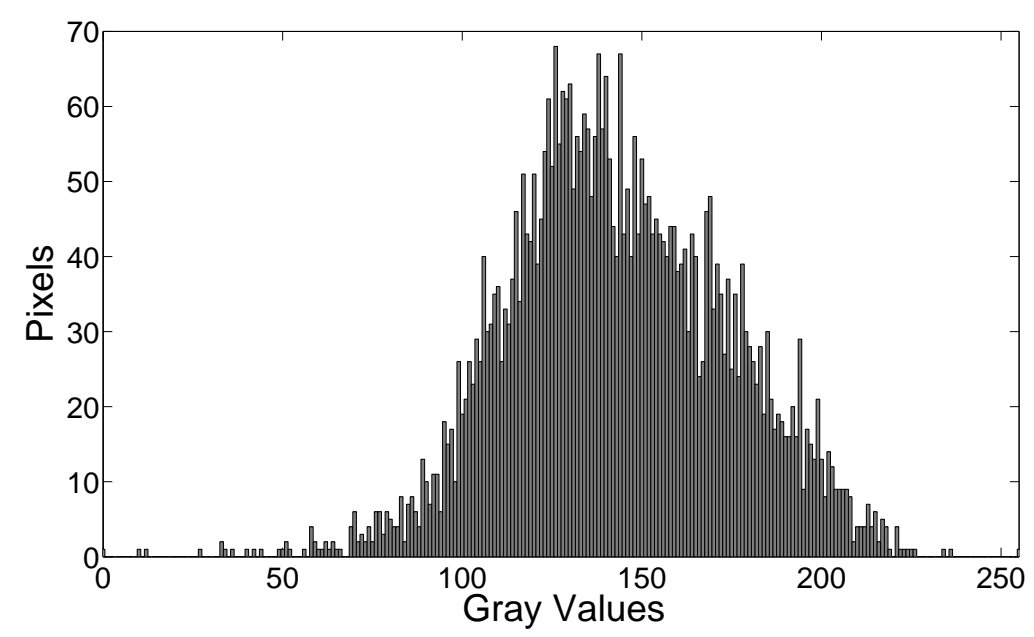

(f)

Figure 5. (a) Skin patch from café-au-lait spot, (b) Obtained source spectra, (c) Melanin quantification, (d) Histogram of melanin quantification, (e) Haemoglobin quantification, (f) Histogram of haemoglobin quantification 